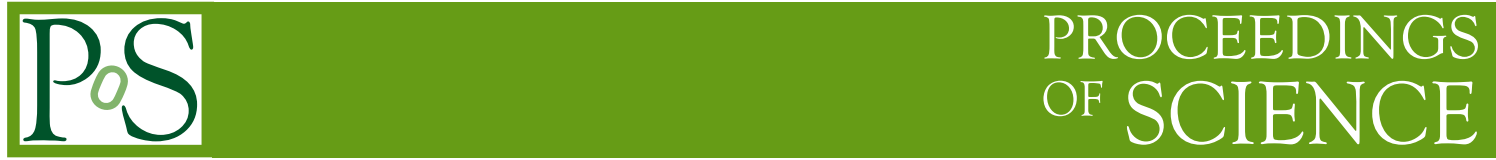

\title{
Thursday question and answer transcripts
}

\section{Thomas A. Trainor*}

CENPA 354290, University of Washington, Seattle, WA 98195 USA

E-mail: trainor@hausdorf.npl.washington.edu

This is a transcript of the question-answer intervals following the Thursday talk presentations. In some cases notes were added during participant review of the transcript (sources are specified). Participants were: Rene Belwied, Kevin Dusling, Ahmed Hamed, Ulrich Heinz, Rudy Hwa, Jiangyong Jia, David Kettler, Che-Ming Ko, Boris Kopeliovich, Christina Markert, Guy Moore, Duncan Prindle, Jan Rak, Lanny Ray, Thorsten Renk, Lijuan Ruan, Edward Shuryak, Raimond Snellings, Mike Tannenbaum, Derek Teaney, Tom Trainor.

Workshop on Critical Examination of RHIC Paradigms - CERP2010

April 14-17, 2010

Austin Texas USA

${ }^{*}$ Secretary. 


\section{Ulrich Heinz}

Mike: Can you show the best data for $v_{2} / \varepsilon$, say up to RHIC, and give your predictions for LHC?

Ulrich: I predict that it will turn over and will be something like $15 \%$ higher at the LHC. Wit Busza would instead say that he expects it to keep increasing roughly linearly to about $50-60 \%$ larger values than at RHIC.

Rene: I'm still trying to understand the quantitative reasoning for how the hadron mass imprints on the $v_{2}$ at low $p_{t}$, and when I can learn something from that fact about how long I'm in the partonic phase and how long I'm in the hadronic phase. Because, you're making this argument that this is just thermal equilibration that gives you the mass splitting. If it's thermal equilibration it's thermal equilibration of hadrons. [yes?] It's not thermal equilibration of partons. [Edward: That doesn't matter. It is a cell which moves with velocity $v$. That's all you have to know.] Well, the elliptic flow builds up in the partonic phase.

Ulrich: What builds up in the partonic phase is momentum anisotropy. So, you can discuss this directly on the level of the momentum-energy tensor, and what you find is that you get a different $T_{x x}$ than $T_{y y}$. That is what hydrodynamics builds up. Now, this gets translated into elliptic flow by converting the energy density from the energy-momentum tensor into particles. If you want to do it early those particles will be partons. If you do it later, at the end of the [nucleus-nucleus] collision, those particles will be hadrons. And how the momentum anisotropy that is encoded in the $T_{\mu \nu}$ manifests itself in an anisotropy of the momentum spectra of the particles depends on the masses of the particles, on their relative chemical composition, how you distribute the overall momentum anisotropy over different particles, all of that matters. And that changes in the hadronic phase as you go down in temperature.

Rene: But we're making an argument that you see at the higher $p_{t}$, where hydro breaks down, you see nice 2 to 3 scaling which you would expect from baryons and mesons. Why don't I see that 2 to 3 scaling, if most of the elliptic flow builds up in the partonic phase, even at low momentum? Why does this translate into a hadron mass [scaling] if I pretend there is no buildup of elliptic flow in the hadronic phase?

Ulrich: No more momentum anisotropy [in the hadronic phase]. You're still changing the elliptic flow in the hadronic phase, but you don't change the momentum anisotropy any more. OK, at RHIC that's not quite true. At RHIC you still increase the momentum anisotropy a bit in the hadronic phase, because by the time the fireball hadronizes it has lost only about half of its spatial eccentricity. So, it's still out-of-plane eccentric and there are still pressure gradients that are anisotropic. So, the hadronic phase, if it were an ideal fluid, would still build more momentum anisotropy. The viscous hadron gas doesn't do it. It builds very little additional momentum anisotropy. But even the viscous hadron gas changes the $v_{2}$, because it redistributes the momentum anisotropy that's already there over the hadrons in a time-dependent fashion because the chemistry and momentum distributions change. You still build radial flow. The spectra get flatter, and so on.

Edward: At least let's first think pure hydro scenario. The medium is locally equilibrated. Any cell has the [???] distribution in [???]. The only reason there is $v_{2}$ is that there are more flowing in one direct than the other. And we don't care which moment it comes. All these ideas are completely irrelevant in this scenario. If you have perfect equilibrium forget it. The people 
who do recombination, who try to derive the formula that $v_{2}$ is increasing by $n^{3}$, assume a different distribution. They assume that you have locally anisotropy in the distribution of particles, which is proportional to $v_{2}$. And if that would be true that is not "flow." That is local anisotropy of distribution of particles in any cell. So you may say "does this effect exist?" In hydro it exists and is proportional to viscosity. I think Derek will be discussing this later. Viscosity is small, it's a very small effect. So in first approximation I'm saying you shouldn't think about where it comes, what is the distribution of particles. You have pieces of matter which fly with different velocities.

Rene: Are the pieces of matter hadrons or partons? Is the fluid cell describing a parton or a hadron?

Edward: You would have to know its velocity and then you know everything. Everything else is just nonsense. More precisely, it is a very small effect proportional to viscosity.

\section{David Kettler}

Rene: If you say that the $v_{2}\{2\}$ that this measures is affected by the same-side jet, you say that essentially the difference between $v_{2}\{2\}$ and $v 2\{4\}$ is the same-side jet peak that you see. But the same-side jet peak is asymmetric. It's not there on the away side. So, why don't you just take the away side and look at what the actual $v_{2}\{2\}$ is?

David: That sounds like a nice idea but it doesn't really work because you still have a dipole [back-to-back jets] on the away side. That's not an issue if you are fitting the whole [azimuth] range. If you're only fitting $\pi$ of that range [away-side interval], actually they [quadrupole and dipole] are not orthogonal Fourier components. They're only orthogonal over $2 \pi$. So the dipole becomes an issue [makes a jet contribution to $v_{2}\{2\}$ only if the fit is restricted to the away side].

Rudy: From this summary there is no mention of hydro. I thought your point is to say that you have something negative to say about hydro.

Thorsten: He's been bashed for this so many times he doesn't dare. [laughter]

David: I don't have anything specific to say about hydro. I just want to present some measurements and interesting trends.

Tom: You may go back to p. 26, and there is implicit in that a statement about hydro, right?

Raimond: Please go to slide 22. This has new insight into the structure of $v_{2}\left(p_{t}\right)$. Because, I mean that's really dramatic. [laughter, silence] Basically, here you describe $v_{2}\left(p_{t}\right)$ of the quadrupole term, right? How does that agree with $v_{2}\{4\}$ or PHENIX or PHOBOS results on the $p_{t}$ dependence?

David: From this centrality [0-5\%] I haven't seen any $v_{2}\{4\}$ results, because they don't do well for the most-central bin. [Edward: It is because it is most central]. For the $p_{t}$-integrated results I compared to $v_{2}\{4\}$, and that only goes up to 10-20\% central. That was the published data.

Raimond: OK, but in the evolution how does that work out if you go to where the $\left[v_{2}\{4\}\right]$ measurements are? It would be quite important to see if that gives the same answer, right?

Tom: There is a full parametrization.

David: Yes. At the end of the talk I gave a complete parametrization of this. I had to kind of skip over it. But it can be compared to any results you want. 
Edward: It is completely correct logic: you go to most central collisions, there is no elliptic flow and what remains is other correlations, for example jets or fluctuations, and they [???] are very small.

Raimond: But, would you agree that in the method you propose you sort of have to at least keep the fluctuations in $v_{2}$ under control, right? Because if for any reason $v_{2}$ would fluctuate a lot you would be sensitive to this.

David: Right, if there were large fluctuations that would contribute to the quadrupole trend. It would make it larger. But we measure a quadrupole term that is basically zero. So, that implies that there are not large fluctuations.

Edward: Well, if quadrupole term is zero by the way...ah quadrupole...you mean $v_{4}$ ?

Boris: No, $v_{2}$.

Ulrich: Can we come back to this plot where you have this horizontal boost on $y_{t}$ ? When you take any single-particle spectrum that is finite at the origin, at $p_{t}=0$ so $\left(1 / p_{t}\right) d n / d p_{t}$ is finite at the origin, you can prove that $v_{2}$ has to go quadratically with $p_{t}$ near $p_{t}=0$. That means $v_{2} / p_{t}$ has to go linearly. So, when I see this solid curve that has a horizontal offset I wonder how you see it.

Tom: The question is, where does the solid curve come from?

Ulrich: What does the single-particle spectrum that corresponds to that black curve look like? I don't think there is a theory that can generate that.

David: That could be, but this is based on the data.

Ulrich: Oh, so it's just some parameters and....

Tom: It's from the quadrupole spectrum [see that page of the talk]. You Uli have some assumptions in mind. In this left panel [quadrupole spectrum page] what you can't quite see, up to the left, those dotted curves are the single-particle spectra, with no boost. The hypothesis here is that the quadrupole is coming from a separate phenomenon with its own spectrum which is boosted by $\left[\Delta y_{t 0}=\right] 0.6$. It's not what you assume in hydro. And that's why there is this...

Ulrich: I'm sorry, $v_{2}$ is an azimuthal Fourier moment of the single-particle spectrum.

Tom: That's what you believe. [laughter]

Ulrich: No, this is how it's measured.

Tom: You are imposing a specific model. You said "it's [something] of the single-particle spectrum, only one spectrum. I'm saying there are two single-particle spectra. One is the thing that starts at zero, and that describes most of the particles. And another is from a separate phenomenon, which exhibits the quadrupole azimuthal asymmetry.

Ulrich: But sorry, $v_{2}$ is defined by the community as the $\phi$ azimuthal second moment of the particle distribution. And I'm saying if that single-particle distribution is finite at the origin this coefficient has to go as $p_{t}^{2}$ at the origin.

Tom: Again, you are using language which assumes a single distribution. There are two superposed distributions. One of them has no...[loud comments]

Edward: There is only one distribution. How do they find two?

Rene: Is there any model that goes with that, any explanation, or is it just a mathematical construct.

Tom: Well, go back to p. 26. What the data tell you, without inserting anything [model assumptions], in the middle panel what the data tell you is that there are some particles in the final state that come from a boosted source, and they have their own spectrum. 
Ulrich: How does that tell you that? Can you show me how you calculate a boosted source from that solid curve?

Tom: That middle panel represents an integral equation-I'm just talking about the blastwave model-it's an integral equation which folds a boost distribution (unknown) with a boosted spectrum. That's the blast-wave model. Taking these data you could in principle invert the integral equation and infer the boost distribution.

Ulrich: But Tom, that model has a $v_{2} / p_{t}$ that goes all the way to $y_{t}=0$, so where does the solid curve go when it runs across it? [Tom: who says?] Because that's what you said. You had a blast-wave model and I know what blast-wave models do.

Tom: I'm talking about a blast-wave model with an arbitrary boost distribution which is to be inferred from the data. You are assuming a certain boost distribution consistent with Hubble expansion [of a bulk medium].

Ulrich: OK, so tell me what the boost distribution is [from the data].

Tom: The boost distribution is, according to these data, [approximately] a delta function corresponding to rapidity $\left[\Delta y_{t}=\right] 0.6$.

Ulrich: And that does not give this black line, I'm sorry. That gives a line that eventually ends up at zero. It may go negative and then go back to zero.

Tom: That's just how the black curve was calculated.

Ulrich: It doesn't stop there. What does it do after it crosses the zero line?

Tom: It does go negative.

Ulrich: And then it goes back to zero, right?

Tom: Right. And you know why it goes negative, because...

Ulrich: Because the spectrum does this...it has a shoulder, it first rises before it goes down [slope changes sign], and therefore you get a negative $v_{2}$ at low $p_{t}$.

Tom: This is just a mathematical representation of what [Sergei] Voloshin argued by words. The point being that there is a rather narrow boost distribution, and in principle it could be a delta function at 0.6 . It would give the negative undershoot. That's a very simple consequence...

Rene: That's a common boost? Shouldn't the curve scale with mass?

Tom: That's taken out by the rapidity. That's why you calculate a rapidity. The rapidity with proper mass is a velocity measure.

Rene: If it's taken out by the rapidity shouldn't they fall on top of each other $\left[v_{2} / p_{t}\right.$ for different hadron species]?

Tom: They do, at the leading edge [of the curves].

Rene: No, I mean the black one and open ones [points] in the middle panel...

Tom: No, because the widths...when you plot on rapidity the widths of the distributions [spectra] go as $T / m$.

Rene: But you take them out you said, when you take transverse rapidity.

Tom: No. The common boost is accommodated. They all line up at the beginning [left edges]. Go back to the quadrupole spectrum [to David].

Rene: Oh, so you're saying that that's where I should see the scaling [at the leading edge]...

Tom: If you plot on boost [rapidity] you see a common source, and the left [spectrum] edges are aligned. For a common temperature $T$, if that is meaningful, then the widths go as $T / \mathrm{m}$ on rapidity. That is just what you see. So, in other words, every feature of this plot [center panel] 
is described by a) the relative abundances of the three hadron species according to the statistical model, b) the widths according to a common $T$ (not due to thermalization $\leftrightarrow$ detailed balance, just quantum mechanics $\leftrightarrow$ phase space) and c) the common boost [distribution]. That is all the moving parts in this $\left[v_{2}\left(p_{t}\right)\right]$. Now you go to the quark-number scaling [right panel, on $m_{t}-m_{0}$ ] and you say "I know exactly why that's that way [quark coalescence]." But it has nothing to do with quark scaling. It's an accident.

Rene: So, this is proof of thermalization. [laughter]

Ulrich: Until you show me a negative data point down here I don't believe it. There's a much more reasonable model...

Tom: There is a published paper, the curve is in a published paper.

Ulrich: Unless you provide me with a [negative] data point that actually lies on this curve, I'm not going to believe your exploding shell.

Tom: Those [proton data] are sitting in STAR right now with very high statistics, proton data that show the negative excursion. Note that curve B is Romatschke [recent viscous-hydro calculation, for pions], as David said. Curve A is Derek's curve from the first quasi-viscous calculations, plotted on rapidity, the famous Teaney curve.

Ulrich: Well, blast wave has its serious weaknesses.

Tom: But notice, the important thing is both those theory curves go smoothly to zero-zero [the origin]. And that's strongly contradicted, especially by the proton data.

Edward: So hydro, remember, hydro describes motion up to some $p_{t}$ where there are rare particles. So, hydro is supposed to describe only this part [smaller $p_{t}$ ]. And all this [larger $p_{t}$ ] has nothing to do with hydro. These are jets.

Tom: In this $p_{t}$ plot on the left [conventional $v_{2}\left(p_{t}\right)$ vs $p_{t}$ ] all the stuff you treasure is jammed into a tiny fraction of that plot [lower-left corner].

Edward: But this [tiny fraction on the left - low $p_{t}$ ] is $99 \%$ of particles. You cannot forget this. All this (to the right) has nothing to do with hydro. We did a good job on that [low $\left.p_{t}\right]$. And all this stuff [higher $p_{t}$ ] nobody explained.

Note added (Tom): The description presented by David (e.g. solid curve for pions) simply describes all $v_{2}\left(p_{t}\right)$ data for three hadron species with a single source boost value, for hadron $p_{t}$ from zero up to $6 \mathrm{GeV} / \mathrm{c}$. No jet contribution to $v_{2}\left(p_{t}\right)$ is needed or even allowed over that interval.

Ulrich: Throw away that $1 \%$ of the particles at the beginning of the discussion.

Edward: You think this curve is wrong [Romatschke] - if you look, compared to your curves? It is absolutely correct, where it should be. And all the rest [trends through data] is nonsense. It has nothing to do with hydro. It has to do with particles with huge rapidities which is one particle per thousand of particles in the event. What hydro can we speak about?

Tom: This will certainly be a topic for the discussion session.

Derek: And dividing zero by zero? $v_{2}\left(p_{t}\right) / p_{t}$ ? And then we see that it [hydro theory curves] disagrees with data? Well, you know, zero divided by zero...

\section{Edward Shuryak}

Boris: So, you have these electric long tubes, and so what? How do you make the ridge? 
Edward: Well, you were late in the first couple of minutes. So, you create this tube, and then there is hydro flow. Hydro flow goes radially from central fireball and it pushes it [tube] with large velocity. Then you see the ridge. The ridge means it is extended in rapidity and it is narrow angle in $\phi$.

Boris: But I want to produce a ridge at high $p_{t}$.

Edward: Particles in the ridge are medium to zero $p_{t}$.

Boris: There are data at several GeV/c $p_{t}$.

Edward: No, that was the trigger. There is a jet trigger that is many GeV/c.

Boris: And associated particles with up to the trigger, right?

Edward: OK, that was in the beginning of my talk. I explain to you later.

Jan: What is your impression for LHC?

Edward: Well, I made a prediction going down in energy, which will happen soon [at RHIC]. At LHC we still have corona, we still have this mixed phase, it has moved a little bit more in the periphery. Since we only see the very peripheral, because of this [opaque] hydro phase, I would think it [the ridge] will also be there. The most interesting thing is what happens when you go down [in energy], particularly with the cone and ridges and all these structures, [is the question] where do they die? In principle, everything is associated with this near- $T_{c}$ region. We can calculate as a function of energy the spatial distribution of matter from hydro, etc. So, we know in detail where the mixed phase is. One can in principle from this idea calculate exactly how they [vary???] with energy.

\section{Duncan Prindle}

Edward: A question about the next to last slide. I understand that you see the jet. And it's remarkable that the jet structure goes down to below $1 \mathrm{GeV} / \mathrm{c}$. How do you see that all partons are accounted for? Where does that statement come from? Because some of them will go from the periphery of the system for sure. How do you know that all of them are coming?

Duncan: What I was trying to say is that the dipole [away-side jet peak] and the same-side Gaussian [jet] peak follow each other [over all centrality, same for p-p and central Au-Au]. You're trying to ask how do I know that all..., how does the same-side Gaussian account for all partons?

Edward: You say that somehow all minijets which are created survive and give you this structure.

Duncan: What I am saying is that for all minijets on the same side there is a partner on the away side.

Edward: All that you see have partners, that is your statement.

Duncan: That's what I'm staying. I think that Tom is making a stronger statement.

Rene: Can you show in this analysis that the away-side integral is equal to the same-side integral? If you say all accounted for it's essentially a momentum-conservation argument, right? So, if you take all the momentum on the away side and compare to all the momentum on the same side you should get equivalent.

Duncan: Well, we have not gotten the momentum.

Rene: You have $p_{t}$ correlations. 
Duncan: We have them. We haven't fit them yet. The shapes are a little bit different. They don't take the same parametrization. So, I don't have the momentum balance. And I think that depends on how broad [on $\eta$ ] things get. Some of this [jet structure] falls outside the acceptance.

Note added (Tom): Only a fraction of jets have partners in a limited $\eta$ acceptance. For one unit of $\eta$ for example the fraction of jet partners is about $10 \%$. One-to-one momentum balance of jets is thus not expected within a limited $\eta$ acceptance. The important point is that the fraction of observed jets with partners does not change from p-p to central Au-Au, is not reduced by absorption in a dense medium in more-central A-A as would be expected for an "opaque core."

Rudy: Your minijets have a [mean] transverse momentum around $1.3 \mathrm{GeV} / \mathrm{c}$ or so. My question has to do with what gives that scale. You show nothing that gives a particular value of $p_{t}$, but you get that from your $y_{t}$ consideration where you cut off the low side because you take off the soft component and it drops fast on the hard component because it's $\log \left(p_{t}\right)$. So, you naturally get a peak associated with a very characteristic $p_{t}$. So, where did that $p_{t}$ come from?

Note added (Tom): The low side is not "cut off" in the minimum-bias $y_{t} \times y_{t}$ plots. The $2 \mathrm{D}$ peak in those plots, interpreted as minijets, is a natural consequence of parton fragmentation. The 2D peak mode (most-probable point) is consistent with the mode $(\sim 1 \mathrm{GeV} / \mathrm{c})$ of the $1 \mathrm{D}$ spectrum hard component in p-p collisions. The latter is simply the projection of the former.

Duncan: From fragmentation of the partons.

Rudy: Yes, but is $1.2 \mathrm{GeV} / \mathrm{c}$ special?

Duncan: The [most probable] parton is more like $3 \mathrm{GeV}$. The argument was that the initial [parton, PDF] state has more partons at even lower [energy] but at some point [below $3 \mathrm{GeV}$ ] you don't have a [hadronic] final state to go to. So, whether the [parton] scattering [to hadrons] occurs or not, it can't occur below some parton momentum, there's no final [hadron] state available. So, the tradeoff between those two constraints happens to result in $3 \mathrm{GeV}$ most-probable parton energy [and $\sim 1 \mathrm{GeV} / \mathrm{c}$ most-probable hadron momentum].

Jiangyong: You call this minijet fragmentation and modified fragmentation. My question is how can you, instead of calling this modification of minijets, distinguish this from the medium response to the minijets? In either case they will always preserve correlations with the original seed that you put in. You can call that modified fragmentation, but the implication is very different, right? All the models that involve medium response, like Mach cones or initial fluctuations (or jets) coupled with radial flow, all have this correlation. If you do a two-component decomposition you will always extract the second component which could have large multiplicity. But it may not be coming from direct fragmentation, because energy conservation will limit the multiplicity. But if you have a re-interaction with the medium, in principle the multiplicity can be very large.

Duncan: I don't think I actually said anything about modified fragmentation. I said "deformation."

Jiangyong: I combined to what Tom Trainor said, and alternative interpretation. My question is, do you have a direct way to separate, to distinguish these two?

Duncan: I don't think I have. There is something going on in the system. The null hypothesis is that...

Jiangyong: If you call modified fragmentation it implies this is not thermalized. If you call it medium response there is some certain level of thermalization. 
Rene: You can't call it jet deformation because your scale changes completely. What you have in a peripheral collision is much smaller than what you have in a central collision. It's not the same minijets. It has to be modified. If you integrate, your amplitude times width times width [same-side peak volume] is much much bigger in central collisions than in peripheral collisions.

Duncan: Around the transition it's not a tremendous difference.

Rene: Right, it doesn't only deform, it also increases tremendously at the same time when it's deformed. It could very well be a different magnitude.

Tom: Remember this is a pair number. If you convert this to fragment number the inferred jet [fragment] multiplicity increases from 2 to 6 [p-p to central Au-Au]. It's not that big an effect. Don't be distracted by the fact that these are pairs. This [peak volume] is going as the square of the fragmentation process.

Christina: You can also produce more particles in a jet with interactions.

Tom: In fact, the jet [fragment] multiplicity in p-p is too small compared to LEP systematics. In some sense this increase [of minijet peak volume] is restoring what was missing in $p-p$ anomalously. There is a strong suppression [of fragment yield] in p-p.

Duncan: One thing I forgot to point out is that maximum amplitudes in our units are a little less that 0.3 . For the same [centrality] bin the dipole is about 0.25 . So, they're about the same size. You look at these plots and you may be tempted to think that's all $v_{2}$. But that's about $50 \% v_{2}$ and the rest away-side jet peak.

Jan: You also mentioned $k_{t}$ broadening. Do you have any numbers?

Duncan: I glossed over that. $k_{t}$ broadening is these things here.

Tom: Can you read for him what the bands are? You [Jan] know very well it depends on your definition of $k_{t}$.

Jan: Right. In this previous slide you mentioned that you don't assume correlation between individual $k_{t 1}$ and $k_{t 2}$, right? In our picture [with Mike Tannenbaum for years] we assumed that $k_{t}$ comes from the Lorentz boost of the pair due to the extra radiation. So, if it's Lorentz boost then the two $k_{t}$ s are strictly correlated. You don't have that assumption, right?

Duncan: In my description I assume they are random [uncorrelated].

Jan: Because, in the Fermi motion picture you would expect they might be uncorrelated. It would be nice to see if we can somehow [distinguish the two cases].

Duncan: If they were completely correlated you would expect to see either this or this [referring to slide on "The other $k_{t}$ broadening"].

Jan: Right.

Duncan: Actually, is that true?

Tom: I don't think it would be any different than this [what is on the slide].

Duncan: It's random event to event. If the $k_{t}$ are correlated to each other for a particular scattering but random relative to the momentum transfer you will get exactly this result.

Note added (Tom): The degree of correlation between the $k_{t} \mathrm{~s}$ of a scattered-parton pair would influence the absolute magnitude of the broadening, not the qualitative features.

\section{Derek Teaney}

Ahmed: I think I would follow earlier and possibly be convinced about hydro. I think this 
would be the first time, just because of the basic question that you started from. Could you please explain why you would start [evolving hydro] around $0.6 \mathrm{fm} / \mathrm{c}$ ? I will not ask about more than $1 \mathrm{fm} / \mathrm{c}$. But what will happen if you start from 0.1 or $0.2 \mathrm{fm} / \mathrm{c}$ ? Can you prove, from the basic principles you start from, that we should start some time about that point $[0.6 \mathrm{fm} / \mathrm{c}]$ with the hydro?

Derek: You want to be starting long compared to $\Lambda_{Q C D} \sim 1 \mathrm{fm} / \mathrm{c}$. You sort of push it and start near there. And, since I do think the [energy???] scale is somewhat larger, it's reasonable to start the decoherence time at $0.6 \mathrm{fm} / \mathrm{c}$.

Ulrich: I think the question went in a different direction: Why is there no flow developing before $0.6 \mathrm{fm} / \mathrm{c}$ ? And the answer is "yes there is." But you cannot describe it by viscous hydrodynamics. Really, what you should do is have a microscopic theory that describes the amount of flow created at early times and then match it to hydro. You cannot do the matching before the [hydro] formalism becomes valid. And, as [Derek] pointed out, if $\eta / s$ is of the order 0.3 or 0.2 you will probably have to wait for about $1 \mathrm{fm} / \mathrm{c}$ before we can continue to propagate with viscous hydro.

Ahmed: Even if you multiply the energy density from the initial energy $\varepsilon_{0}$ by a factor 4 or 5 ?

Derek: It doesn't matter. What matters is the $1 / \tau$.

Ahmed: Then there is a very important point here. We all know that $\alpha_{s} \ldots$ it is $\Lambda_{Q C D}$ for the vacuum [???], to $150 \mathrm{MeV}$ maybe. Now, if you compress the matter, $\alpha_{s}$ for sure would change. We don't know what would be the scale for QCD there. Then how does it come? We can use the energy density and you still need that $0.6 \mathrm{fm} / \mathrm{c}$.

Derek: You don't need $0.6 \mathrm{fm} / \mathrm{c}$ - for sure not. Most of the transverse dynamics happens on a longer time scale, like $3 \mathrm{fm} / \mathrm{c}$. So, you don't need to start at $0.6 \mathrm{fm} / \mathrm{c}$.

Edward: The answer in one sentence is hydro itself tells when we can start. [???] will tell you the time and the temperature you can use it.

Rene: Equilibrium still has to be an issue, right? Is it equilibrated or not?

Derek: We assume that we're approximately equilibrated. It doesn't need to be perfectly equilibrated. But if we look at one of the plots that Uli showed was that if you changed the initialization of $\Pi$ [momentum flux density???] you find it. It relaxes on a time scale of $1 /(\eta / s)$, and the final results are independent of what you take for $\Pi$, and that's what we find too. The precise way you initialize leads to $\eta / s$ sufficiently small.

Rudy: Let me ask the question in a different way. Suppose you fix $\eta / s$ at 0.2 and plot the result as a function of $\tau_{0}$. How much of a range do you...

Derek: Very little.

Rudy: It would be nice to show that.

Derek: Because you're sort of...we've made plots. You're going backwards in time... and you're looking at the transverse dynamics which develops over a longer time scale. So, in that short time [interval] you get basically Bjorken expansion and not too much else.

Ulrich: I think your answer is not quite correct (except for ideal hydrodynamics). I think that in viscous hydrodynamics when you go to earlier times you have viscous entropy production. You have to renormalize your entropy so that you still get the same final multiplicity. And in the end you do get more radial flow when you start earlier.

Derek: I guess the good thing about the viscous [hydro] is that it tells you when you should start. You have $\eta / s$. If you go back too early, you'll see that your gradients are huge compared to the scale you want to evolve it on, which just tells you your doing something wrong. 


\section{Lanny Ray}

Mike: I believe most of the stuff you said, but I didn't see $p_{t}$ spectra of these so-called jets.

Lanny: I believe that was [shown] on the first day.

Mike: Well, what is it? Goes like what, $p_{t}$ to a power? Where is it? How does it compare to QCD?

Lanny: I have it on a backup. Here it is.

Mike: Thanks. [Lanny: Does that help?] No. [laughter] Just straight out: You say you have jets, whatever you call it. You have a cone, a nice jet correlation. You say they're jets. What's the $p_{t}$ spectrum? Even Rubia showed a $p_{t}$ spectrum.

Tom: You're asking effectively for the parton $p_{t}$ spectrum?

Mike: No. What you see, whatever you call it. You have an object.

Tom: Well, this is by hypothesis the fragment $p_{t}$ spectrum. Are you asking about the parent parton $p_{t}$ spectrum?

Mike: You have some blah, that looks like a cone.

Tom: The answer is we have both. We have all of these [???] on a semilog plot.

Mike: OK, that's the two-particle correlations. What's the p-perp?

[obscured by background conversation]

Mike: OK, you see jets by two-particle correlations. So what else is new?

Rene: I just want to make one comment to the radial expansion models. I don't think it's quite true to say they do not describe the growth on the away-side ridge. The local momentum conservation term is in these models. The local momentum conservation grows when the ridge on the same side...

Lanny: So, ...

Rene: You look at the Brazilian paper...is just a more quantitative thing...

Lanny: I have yet to figure out what's in NEXSPHERIO [Brazilian paper], as far as what produces its away-side correlation, and I have not gotten a straight answer...

Edward: The authors themselves have to figure out what is happening inside their code. I spoke with them about it. It's still a mystery how they get it.

Rene: How they get the away side?

Edward: Yes.

Lanny: I have asked that several times and gotten [no answer].

Rene: I was also under the impression that it's the momentum conservation term...

Thorsten: If you have a near-side ridge there must be something balancing on the away side.

Rene: The momentum has to be balanced.

Edward: Somehow, I find it a bit strange that there is some philosophical difference between your findings and the kind of framework you looked at this. You find that there are some transitions in this phenomenon. And you have evidence which is very interesting that there is strong energy dependence-62 and $200[\mathrm{GeV}]$ are different. And the positions where they [transitions] happen seem to correspond to the same density of matter. And then you look for solutions in pQCD. pQCD doesn't care about specific density of matter. It's everything proportional to density, a trivial dependence. Is some transitions in medium...

Boris: Is not true, without coherence maybe but with coherence not true. 
Edward: OK, then have two formulas, proportional to $n$ and $n^{2}$.

Boris: No, no. QCD does have coherence above proportional to density.

Edward: Yes, but it doesn't have phase transitions as a function of density. It's a many-body phenomenon, I'm saying. You will never understand it in pQCD. It's a phenomenon in medium. It is clear that it is a function of density, from your [Lanny's] findings.

Tom: Isn't that Lanny's last point though. You're talking about pQCD in a particular context. Now we have...

Edward: I never understand what is coherent there. I know in the parton model it is incoherent collision of partons. Everything else is a mystery to me.

Boris: There is Landau-Pomeranchuk, which is different from what you say.

Edward: It's just modification of jet quenching. We're not speaking about jet quenching.

Yuri: It is not modification of jet quenching, it is a new mechanism for producing partons. It's not about energy loss, it's about...

Boris: I'm talking about Landau-Pomeranchuk in the initial conditions, not in the final state.

Derek: How does the growth of the [eta] width of the [same-side] ridge [jet peak], $\left[\sigma_{\eta}\right]$ how does that depend on the multiplicity? You found a sudden transition of this width, and the multiplicity $d n / d \eta$ is smooth there? What happens with that?

Lanny: Do you see something corresponding in the multiplicity? [yes] It's really hard to see. The question was: near the transition do we see a similar glitch in the multiplicity vs $v$ ?

Tom: Yes. In fact I showed that in one of my last few slides. I was using the two-particle [sameside jet] correlations to infer single-particle yields. Where this transition occurs the measurements of total particle multiplicity are not very well established. Because that's actually quite peripheral collisions. So, if you plot the same thing on $n_{\text {part }}$ [participant number] where the transition occurs is rather small $n_{\text {part }}$. Measurements there at RHIC are unfortunately rather sparse and poor.

Lanny: So, the answer is yes, that's something that in principle can be looked at. What about $62 \mathrm{GeV}$ ?

Tom: Same problem for $62 \mathrm{GeV}$.

Lanny: So, I think that's a criticism of the lack of precision data.

Tom: It's a cultural issue, because the concentration...

Lanny: In fact Lijuan [STAR spectra working group convener], not to pick on you, but you're representing all things spectra. Do we have precise measurements of $n_{c h}$ vs centrality, especially for $\mathrm{Au}-\mathrm{Au}$ at $62 \mathrm{GeV}$ ? We have what we just published, but that's coarse bites.

Lijuan: We just published the data, and basically [????]. For global multiplicity as a function of centrality we do not see the transition. It's quite smooth.

Lanny: So, that's the answer, with the given statistics we have we don't see it.

Note added: At the transition point the fraction of multiplicity coming from fragmentation (hard component) is significantly less than $10 \%$ of the total, so unambiguous observation of the transition with $d n_{c h} / d \eta$ would require very accurate data, which are not currently available.

Mike: I have to say there's nothing between $5 \mathrm{GeV}$ and $200 \mathrm{GeV}$.

[obscured by conversation]

???: What's it like in $\mathrm{Cu}-\mathrm{Cu}$

Lanny: It's all over the place. It goes from something like $60 \%$ all the way down to $20 \%$ in copper. 
Jiangyong: But in $n_{\text {part }}$ that's a small number, 50-60. Hydro may not be that fully applicable at that point. The sharp transition may just be the turn-on of radial flow.

Lanny: I don't think hydro is applicable there.

Jiangyong. Hydro works....actually hydro works I would say above $n_{\text {part }}=100$.

Edward: It's not the work of hydro. If you look at $v_{2}$ it doesn't have anything special at these points, special for copper.

Tom: You should point out that conventional practice is to not measure the first two centrality points [on those plots, which establish the linear-superposition baseline]. That's forbidden!

Lanny: Yes. Actually one reason we [STAR] didn't see this for a long time was because typically these first two [centrality] bins [are missing]. Normally, [conventionally] data stop at the $80 \%$ point. Plus, you combine that with plotting on $n_{\text {part }}$ which takes all of this [peripheral region] and jams it down into the corner. So all this part [including the sharp transition] is jammed into the corner.

Tom: That's another example of the choice of plotting format favoring or disfavoring certain assumed mechanisms.

Boris: Let me ask this question. From jet quenching we can extract the transport coefficient. On the other hand we could measure broadening directly would be a serious test. The broadening for away-side jets contains information.

Lanny: Yes, but that's very hard to measure, because that's [away-side peak on azimuth is] already [very broad], at least in the momentum range we are in. As you go up in [fragment, parton] momentum you could do that [what Boris suggested].

Mike: What is the momentum range you're in?

Lanny: We have no momentum cutoff. That's the plot that you don't like.

Mike: You said a momentum range. Just tell me the range.

Lanny: Our lower limit is $0.15 \mathrm{GeV} / \mathrm{c}$. Where this [jet] structures sits is at $1-1.5 \mathrm{GeV} / \mathrm{c}$, say from 0.8 to $2 \mathrm{GeV} / \mathrm{c}$, where most of the [jet fragment] particles contribute.

Tom: Duncan showed the measurements in p-p of the $k_{t}$ broadening evolution with $p_{t}$ cuts. So, that's been done. The $k_{t}$ evolves...it decreases the softer you go because of kinematic constraints. If you choose smaller-momentum hadrons that will correspond to smaller allowed $k_{t}$ [the $k_{t}$ distribution is biased by the hadron selection].

Boris: But this broadening comes from the primordial transverse momentum.

Tom: Yes, in p-p.

Lanny: He's asking something a bit different. As the jet quenches in the medium there is $\hat{q}$ which measures that broadening.

Tom: Then we go back to what Lanny showed. The away-side peak is already so very broad...

Lanny: It might broaden further, but it's still going to look like a dipole.

Rene: You say [there are jets] between 0.8 and $2.0 \mathrm{GeV} / \mathrm{c}$, and you do that on the basis of your $y_{t}$ cuts...

Lanny: Yes, that's on the basis of where that [hard component] bump is on $y_{t}$.

Rene: Do all these plots have the same [vertical] scale? Because the particles below $0.8 \mathrm{GeV} / \mathrm{c}$ you have so many more. My question is, if you now scale the bump itself...if you go to the next plot [???] where you show the [different ytxyt plots???]...

Lanny: [discussion of weighting procedure to get average histograms] 


\section{Kevin Dusling}

Jiangyong: You have this two-component-gluon-quarks-which flow with the same radial flow, but they have different cross sections, so they have different anisotropy before freezeout. Then you assume that gluon goes to baryon dominantly, and quarks go to mesons, right?

Kevin: That was certainly the assumption made in this picture. That's what got us thinking about this meson/baryon scaling. If you make that assumption here you get this beautiful scaling. They're right on top of each other. And that's a pure theory calculation. There is no fine-tuning there. The relaxation time between quarks and gluons came out of QCD. We just chose to scale the gluons by 3 and the quarks by 2 based on the thinking you had, and we saw this. And then we said OK now let's do some phenomenology with this. And then we go to the meson/baryon scaling where we keep this ratio of cross sections arbitrary.

Rene: You are not saying the baryons are made from gluons and the mesons from quarks, your not saying that.

Kevin: We're not saying that.

Jiangyong: But he assumed. Otherwise how do you get the scaling?

Kevin: No. Here we have two different scales related to the relaxation times of mesons and baryons, and that's sort of chosen. This fit parameter $c_{M} / c_{B}$ is the ratio of relaxation times for mesons and baryons.

Rene: It's a fit parameter.

Guy: The picture in this case is, supposing that hadronization happens while you're still in the hydrodynamic regime, and then there's a brief period when your hadrons are hydrodynamic. During that period the departure from the ideal curve is determined by the inverse of the size of your scattering cross section. Now, I have no idea what the relative scattering cross section of a baryon and a meson is, so I'll let it be some number and see what fits the data. And if the number is 1.5 or 1.6 then with one fitting parameter I can get both...

Jiangyong: But this tells me something about hadronization, right?

Kevin: This parameter tells you something about the relative rates of mesons and baryons, the relative cross sections.

Thorsten: Wouldn't that predict different decoupling temperatures? If the baryon has a larger interaction cross section?

Kevin: Probably slightly, yes.

Che-Ming: I'm a bit confused. Ulrich told us most of the $v_{2}, 80 \%$ develops in the partonic state. Now you say all the scaling is from the hadronic state. But Ulrich says $20 \%$ is from the hadronic state.

Kevin: The momentum anisotropy develops during the partonic state.

Thorsten: That's just in the tensor. That's just the fluid element. At some point you need to commit and say I want to convert this into a hadron, and you need to specify how to do it.

Kevin: This momentum anisotropy builds up throughout the whole evolution. Then you finally get to freezeout and you need to produce your particles, and when you do that, Uli and I are doing it the same way, but that happened because of this buildup of anisotropy early on. 